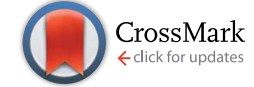

Cite this: RSC Adv., 2017, 7, 1413

Received 16th October 2016 Accepted 14th November 2016

DOI: $10.1039 / c 6 r a 25309 a$

www.rsc.org/advances

\section{High coercivity stellated cobalt metal multipods through solvothermal reduction of cobalt hydroxide nanosheets $\dagger$}

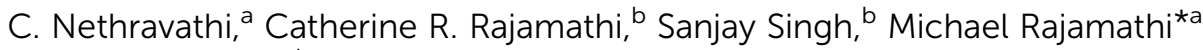 \\ and Claudia Felser ${ }^{\mathrm{b}}$
}

\begin{abstract}
Solvated 2D nanosheets of dodecylsulphate intercalated $\alpha$-cobalt hydroxide in 1-butanol are solvothermally reduced to hexagonal close packed (hcp) Co metal multipods in the presence of oleylamine. 1-Butanol, being a reducing solvent, facilitates the formation of Co metal. In addition to being a reducing agent, oleylamine also acts as a base which converts the metastable $\alpha$-cobalt hydroxide nanosheets into $\beta$ hydroxide sheets. These sheets yield face centered cubic (fcc) $\mathrm{CoO}$ spheres which eventually grow into hcp Co metal multipods. All the pods are single crystalline with growth direction being [001], making the multipods resemble stellated polyhedra with overgrown branches. The stellated Co metal multipods exhibit high coercivity $\left(H_{c}\right)$, of 880 and $552 \mathrm{Oe}$ at $2 \mathrm{~K}$ and $300 \mathrm{~K}$ respectively, due to shape anisotropy.
\end{abstract}

\section{Introduction}

Magnetic nanostructures exhibit size and shape dependent unique magnetic properties. ${ }^{\mathbf{1 - 9}}$ As a result, they are attractive materials in ultra-high density magnetic recording, ${ }^{10-12}$ catalysis $^{13,14}$ sensing $^{15}$ and biomedicine. ${ }^{16}$ Anisotropic nanostructures of magnetic metals are known to exhibit high remanence and coercivity. ${ }^{17}$ This is because the high aspect ratio of anisotropic structure creates a high demagnetizing field. ${ }^{17}$ Nanocubes of Ni exhibit four times enhancement in magnetic saturation in comparison to superparamagnetic spherical $\mathrm{Ni}$ particles of similar size. ${ }^{18}$ Cubic Co nanoskeletons exhibit higher coercivity (145 Oe) compared to Co hollow shells (75 Oe).$^{19} \mathrm{CoPt}$ nanopolypods exhibit a coercivity of 525 Oe at room temperature, much higher compared to CoPt nanowires. ${ }^{20}$

Although well-defined multipods of semiconductor materials (CdS/Se/Te) are well documented, synthesis of multipods of metals like Pt, Pd, Au, Ag and Rh has been challenging due to the high degree of symmetry in the face centered cubic (fcc) crystal structure. ${ }^{\mathbf{2 1 , 2 2}}$ The fact that existence of polymorphism supports the synthesis of branched nanostructures was recently demonstrated in the case of magnetic transition metal, $\mathrm{Ni}$, which is known to crystallize in fcc and hexagonal close packed (hcp) phases. ${ }^{23}$ It should be possible to synthesize multipods of Co, which too exhibits a similar polymorphism. Besides the interesting size-dependent structural, magnetic, electronic and

${ }^{a}$ Materials Research Group, Department of Chemistry, St. Joseph's College, 36 Lalbagh Road, Bangalore 560027, India. E-mail: mikerajamathi@rediffmail.com

${ }^{b}$ Max-Planck Institute for Chemical Physics of Solids, Dresden, Germany

$\uparrow$ Electronic supplementary information (ESI) available: TEM images and magnetic studies. See DOI: 10.1039/c6ra25309a catalytic properties, the exponential dependence of magnetization relaxation time on the volume and shape anisotropy has triggered a lot of interest in the synthesis of cobalt nanostructures for magnetic storage devices. ${ }^{1}$

Multipods or branched metal structures with high surface area are also attractive for sensor based applications and have been shown to exhibit enhanced catalytic/electrocatalytic activity. $^{21}$

Zero dimensional (nanocrystals, ${ }^{1}$ hollow spheres ${ }^{24} /$ particles $^{25}$ ), one dimensional (nanorods, ${ }^{26}$ nanobelts, ${ }^{27}$ nanowires, ${ }^{28}$ nanochains $^{29,30}$ ), two dimensional (nanodisks, ${ }^{31,32}$ nanoplatelets, ${ }^{33}$ superlattices $^{34,35}$ ) and three dimensional (flower-like, ${ }^{36}$ hyperbranched $^{37}$ ) structures of cobalt metal have been synthesized and are known to exhibit unusual intrinsic properties in comparison to bulk. Synthesis and magnetic properties of multipods or highly branched structures of cobalt metal are largely unexplored.

Exfoliation of layered transition metal hydroxides in solvents yields colloidal dispersions of two-dimensional (2D) metal hydroxide nanosheets. ${ }^{38}$ Solvated hydroxide nanosheets can be chemically reacted at room temperature or under solvothermal conditions to yield oxide nanostructures of interesting morphologies. ${ }^{7,39-41}$ Single step reaction, employing solvated hydroxide nanosheets as precursor, results in unusual shapes and interesting properties in contrast to multi-step reactions involving metal salts/complexes and surfactants. In this work, we describe the synthesis of hcp Co metal multipods through solvothermal reduction of solvated 2D nanosheets of dodecylsulphate (DS) intercalated $\alpha$-cobalt hydroxide in the presence of oleylamine. Here, the hcp Co multipods are formed in the absence of fcc Co nuclei as intermediates and these multipods exhibit high shape anisotropy due to high pod density and the high crystallinity of the individual pods. 


\section{Experimental}

2.1 Synthesis of dodecylsulphate intercalated $\alpha$-cobalt hydroxide

$\mathrm{Co}(\mathrm{OH})_{1.67}(\mathrm{DS})_{0.33} \cdot 1.1 \mathrm{H}_{2} \mathrm{O}$, was synthesized as described in our earlier work. ${ }^{42}$ A solution $(35 \mathrm{~mL})$ containing cobalt acetate (1.24 g) and sodium dodecylsulphate, DS (1.44 g) was added to $50 \mathrm{~mL}$ of $0.5 \mathrm{M}$ aqueous $\mathrm{NH}_{3}$ solution with constant stirring. The green precipitate obtained was immediately centrifuged, washed free of anions with water and then with acetone and dried in air at room temperature.

\subsection{Synthesis of Co metal multipods}

A mixture of $40 \mathrm{mg}$ of DS intercalated $\alpha$-cobalt hydroxide and $45 \mathrm{~mL}$ of 1-butanol was subjected to sonication, for $45 \mathrm{~min}$. To the resulting colloidal dispersion, $5 \mathrm{~mL}$ of oleylamine was added and sonication was continued for another $15 \mathrm{~min}$. The dispersion was transferred into a Teflon lined autoclave. The autoclave was sealed and heated at $220{ }^{\circ} \mathrm{C}$ for $24 \mathrm{~h}$. The black solid obtained was washed with ethanol and dried at room temperature under vacuum. In order to observe the intermediate stages, the reaction was carried out for different time durations $-4,8$ and $16 \mathrm{~h}$. As control experiments, the synthesis was repeated (i) in the absence of oleylamine and (ii) with cobalt acetate in the place of DS intercalated $\alpha$-cobalt hydroxide.

\subsection{Characterization}

All the samples were analyzed by recording powder X-ray diffraction (XRD) patterns using PANalytical X'pert pro diffractometer $(\mathrm{Cu} \mathrm{K} \alpha$ radiation, secondary graphite monochromator, scanning rate of $1^{\circ} 2 \theta \mathrm{min}^{-1}$ ). Scanning electron microscopy (SEM) images were recorded on a Hitachi S4800 electron microscope operating at $15 \mathrm{kV}$. Transmission electron microscopy (TEM) images were recorded using a JEOL F3000 microscope operated at $300 \mathrm{kV}$. The isothermal magnetization $[M(H)]$ at $2 \mathrm{~K}$ and $300 \mathrm{~K}$ was measured using a superconducting quantum interference device (SQUID-VSM) magnetometer.

\section{Results and discussion}

A series of $00 l$ reflections in the XRD pattern (Fig. 1a) of the precursor, DS intercalated $\alpha$-cobalt hydroxide, suggests that the layered solid is well ordered along the stacking direction. The basal spacing of $25.8 \AA$, calculated from these reflections, confirms the incorporation of DS anions. DS intercalated $\alpha$-cobalt hydroxide readily exfoliates in 1-butanol, on sonication, resulting in a colloidal dispersion of the hydroxide nanosheets. The absence of $00 l$ reflections and presence of only in plane $2 \mathrm{D}$ reflections at $2 \theta \sim 33^{\circ}$ and $60^{\circ}$ in the XRD pattern of the dispersion (Fig. 1a, inset) indicate that solvation causes the $\alpha$-cobalt hydroxide layers to separate out and behave as independent 2D nanosheets. ${ }^{42}$ The XRD pattern of the black powder, obtained on solvothermal reduction of DS intercalated $\alpha$-cobalt hydroxide nanosheets in the presence of oleylamine (Fig. 1b), could be indexed to pure hcp Co metal (JCPDS no. 5-727).

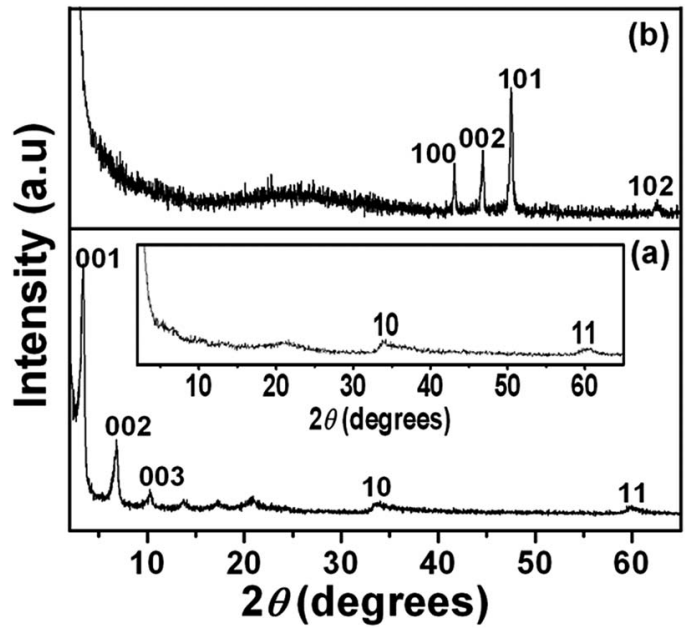

Fig. 1 XRD patterns of DS intercalated $\alpha$-cobalt hydroxide (a) and Co metal obtained on solvothermal reduction (24 h) of colloidal dispersion of DS intercalated $\alpha$-cobalt hydroxide (b). The in situ XRD pattern of the colloidal dispersion of DS intercalated $\alpha$-cobalt hydroxide in 1butanol is shown as inset in (a).

The hcp Co metal obtained on solvothermal reduction of dodecylsulfate intercalated $\alpha$-cobalt hydroxide exhibits a uniform morphology of three dimensional multi-pod assemblies (Fig. 2). Each assembly consists of multiple pods originating from a common core. The mean diameter and length of the pods are $\sim 130 \mathrm{~nm}$ and $\sim 320 \mathrm{~nm}$ respectively. The tips of the hcp Co metal pods are flat and in the shape of hexagons of $\sim 190 \mathrm{~nm}$ width. Though the pods are of uniform dimensions the assemblies vary in the density of pods. Most of the multipods have more than ten pods.

Bright field TEM images of the multipods shown in Fig. 3a and $b$ reveal that neither the pods nor the core is hollow. The selected area diffraction (SAED) patterns of multiple pods (Fig. 3c) and of a single pod (Fig. 3d) suggest that the pods are single crystalline in nature and the growth direction of individual pods in the assemblies could be [001]. The hexagonal facets at the ends of the pods as seen in the SEM image (Fig. 1b) further indicate that the growth direction of the pods is [001]. Thus the multipods resemble stellated polyhedra with the pods overgrown along [001] from the core.

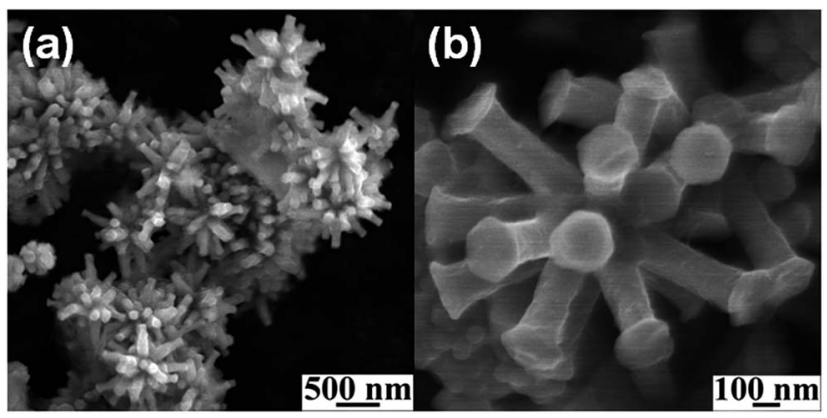

Fig. 2 SEM images of stellated Co metal multipods. 

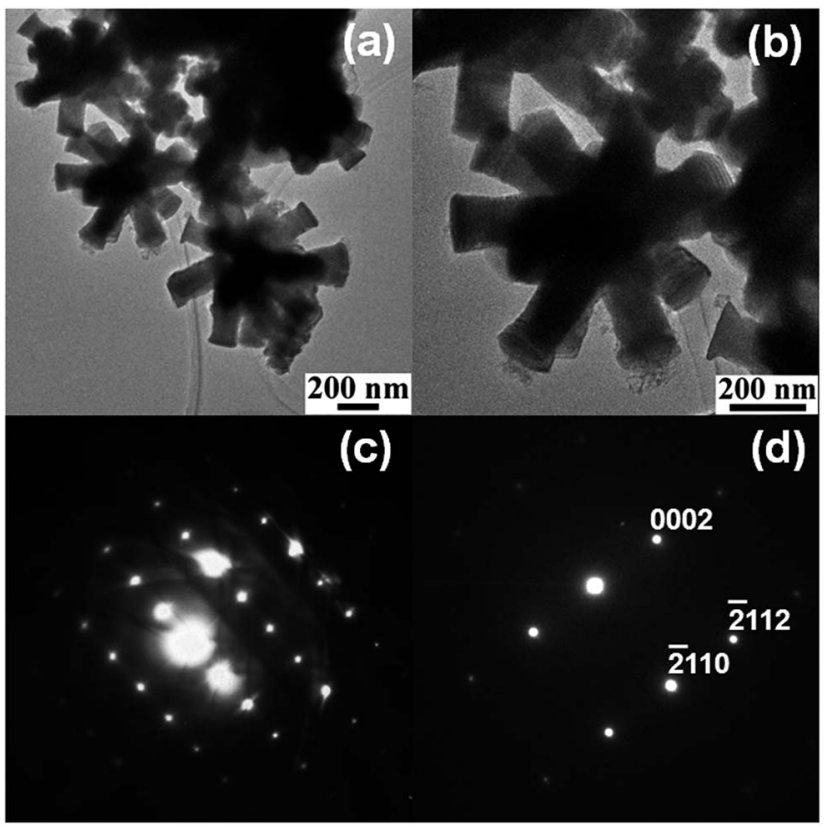

Fig. 3 Low (a) and high (b) magnification bright field TEM images of stellated Co metal multipods and electron diffraction pattern from multiple pods (c) and a single pod (d) of the multipods.

In order to understand the growth mechanism of the stellated Co metal multipods, intermediate structures were isolated by quenching the reaction at different time intervals. The XRD pattern (Fig. 4A(a)) and TEM image (Fig. 4B) suggest that intermediate obtained at the end of $4 \mathrm{~h}$ consists largely of $\beta-\mathrm{Co}(\mathrm{OH})_{2}$ nanosheets. Oleylamine, as a reactant, contributes to the basicity of the reaction mixture thus inducing deintercalation of DS anion of $\alpha$-cobalt hydroxide leading to topotactic transformation of the metastable $\alpha$-cobalt hydroxide nanosheets to more stable $\beta-\mathrm{Co}(\mathrm{OH})_{2}$ nanosheets.

As the reaction proceeds $(8 \mathrm{~h}), \beta-\mathrm{Co}(\mathrm{OH})_{2}$ is decomposed to fcc $\mathrm{CoO}$ (Fig. 4A, b) and during this dehydroxylation step, the thin nanosheets role into spheres of varying sizes, $\sim 200-$ $500 \mathrm{~nm}$ in diameter (Fig. 4C and D). The full width at half maximum (fwhm) of the Bragg reflections in XRD (Fig. 4A(b)) and the diffuse ring electron diffraction (ED) pattern (ESI, Fig. S1†) suggest that these spheres are polycrystalline aggregates of $\mathrm{CoO}$ nanocrystals. The reducing nature of butanol and oleylamine not only support the formation of fcc CoO compared to the more stable spinel $\mathrm{Co}_{3} \mathrm{O}_{4}$ but also facilitates further reduction of fcc $\mathrm{CoO}$ to hcp Co metal (Fig. $4 \mathrm{~A}(\mathrm{c})$ ), as the reaction proceeds further (16 h). TEM images of the intermediate obtained at the end of $16 \mathrm{~h}$ (Fig. $4 \mathrm{E}$ and $\mathrm{F}$ ) reveal that the dense spheres germinate into pods of hcp Co metal in all directions (Fig. 4E and F) thus indicating that these spheres act as nucleating cores. The smaller spheres result in less dense multipod assembly compared to the larger spheres (Fig. 4F).

The control experiment using cobalt acetate salt as precursor under similar experimental conditions results in the formation of spherical flower-like aggregates of thin flakes of fcc Co (ESI, Fig. S2 $\dagger$ ), indicating that using DS intercalated of $\alpha$-cobalt hydroxide nanosheets as precursor seems to be crucial for the

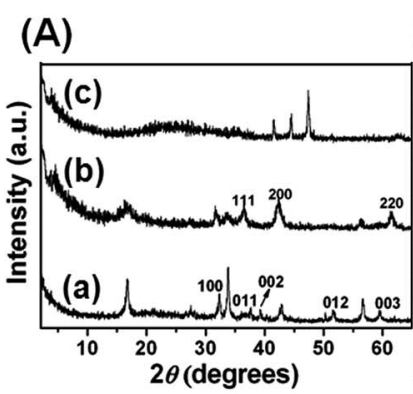

(B)
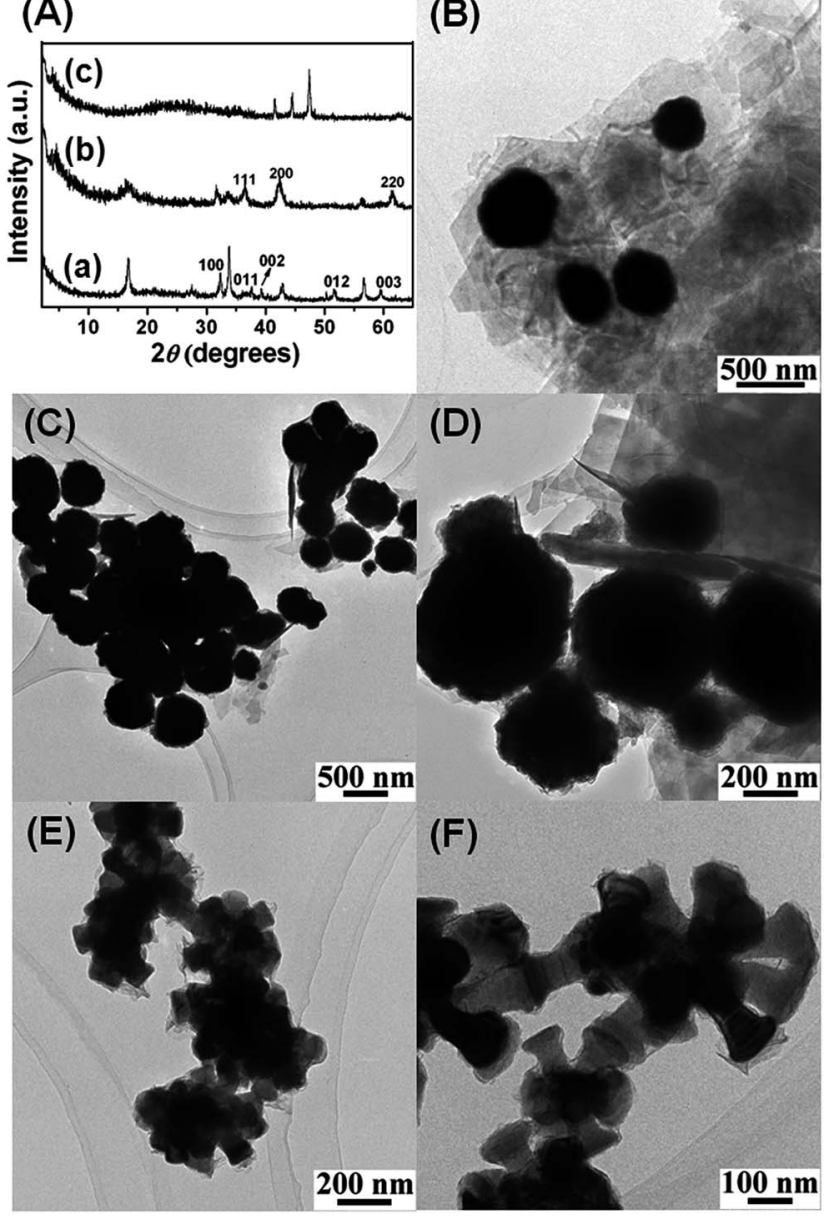

(F)

Fig. 4 (A) XRD patterns of the intermediates obtained on solvothermal decomposition of DS intercalated $\alpha$-cobalt hydroxide in 1-butanol in the presence of oleylamine at the end of 4 (a), 8 (b) and $16 \mathrm{~h}$ (c). Bright field TEM images of the intermediates obtained at the end of $4 \mathrm{~h}(\mathrm{~B})$, $8 \mathrm{~h}(\mathrm{C}$ and $\mathrm{D})$ and $16 \mathrm{~h}(\mathrm{E}$ and $\mathrm{F})$.

formation of hcp Co multipod assemblies. When the reaction was carried out starting from $\alpha$-cobalt hydroxide nanosheets but in the absence of oleylamine there was no conversion and the $\alpha$-hydroxide remained almost intact. This suggests that the basicity and the reducing action of oleylamine are important for the formation of the multipods.

In the absence of the formation of fcc Co nuclei at any stage of the reaction, we may explain the formation of the multipods by a pair of pseudotopotactic transformations. The arrangement of Co atoms in [001] direction of the hexagonal $\beta$-cobalt hydroxide is the same as that of the [111] planes of the cubic $\mathrm{CoO}^{43}$ Thus the hydroxide is converted into the cubic $\mathrm{CoO}$ readily in a pseudotopotactic fashion. During this transformation the erstwhile hydroxide sheets roll up to give $\mathrm{CoO}$ spheres. These polycrystalline spheres are aggregates of $\mathrm{CoO}$ nanocrystals with their [111] facets exposed on the surface of the sphere. The growth of the Co metal pods originates from these [111] facets of CoO. As the arrangement of Co atoms in hcp Co metal in the 001 direction is the same as that of the [111] planes of the cubic $\mathrm{CoO}^{43}$ the growth direction in the pods is 
[001]. The high surface energy of the [111] facets of CoO facilitate the preferential nucleation of hcp Co on these facets. ${ }^{23}$

Magnetization measurements were carried out on the stellated hcp Co multipods as the unique arrangement of these multipods is expected to play an important role on the magnetic properties. The temperature dependence of the magnetization (ESI, Fig. S3†) shows no magnetic transitions in the $2-300 \mathrm{~K}$ temperature range, as pure Co presents a Curie temperature around $1400 \mathrm{~K}$. Magnetic hysteresis loops $[M(H)]$ (Fig. 5a) were measured on the powder
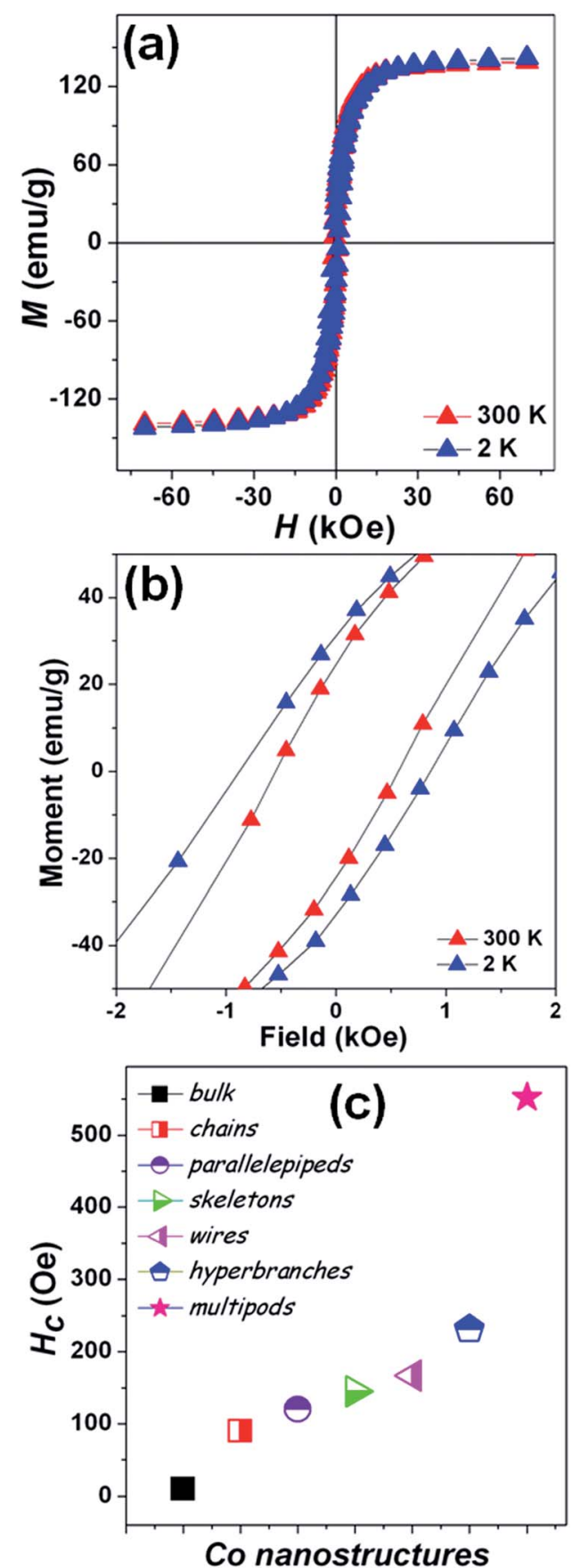

Fig. 5 Magnetic properties of stellated Co multipods. (a) The hysteresis loops of the Co multipods at 2 and 300 K. (b) The comparison of coercive fields at 2 and $300 \mathrm{~K}$. (c) The comparison of the coercive fields for the different cobalt nanostructures with bulk. sample such that the individual stellated multipods are randomly oriented..$^{\mathbf{2 9 4 4 , 4 5}}$ The saturation magnetization $\left(M_{\mathrm{S}}\right)$ at 2 and $300 \mathrm{~K}$ of the stellated Co multipods are 141.9 and $139.2 \mathrm{emu} \mathrm{g}^{-1}$, respectively. The $M_{\mathrm{S}}$ is about $17 \%$ lower than the value reported for Co bulk (168 emu $\left.\mathrm{g}^{-1}\right){ }^{28}$ While the presence of synthesis-related impurities or antiferromagnetic oxides on the surface can never be completely ruled out, ${ }^{\mathbf{4 6 - 4 8}}$ the lower $M_{\mathrm{S}}$ may also be understood as a consequence of this particular multipod arrangement.

Here, each pod is a single crystalline, hexagonal-based pillar, where the long growth axis corresponds to the $001 c$-axis which is the easy magnetization direction in Co. These pods are rigidly attached to a core as to form a radial or stellated structure, where each pod will have their easy magnetization axis along their own growth direction. Due to magnetocrystalline anisotropy, when a magnetic field is applied to the stellated Co multipod, the contribution of each pod to the net magnetization will depend on the angle between the $c$-axis of that pod and the external magnetic field. The larger the angle between a pod's $c$ axis and the applied magnetic field, the larger the component perpendicular to the field of the individual pod magnetization will be, thus lowering the net magnetization. Notice that there is a symmetry point perpendicular to the applied field, i.e. the pods pointing down in relation to the magnetic field will contribute in the same way as those pointing up. The stellated multipod arrangement should also give rise to larger shape anisotropy, resulting in high coercive fields.

This is indeed observed, as the coercive fields (Fig. 5b) of the stellated Co multipods at 2 and $300 \mathrm{~K}$ are 880 and 552 Oe, respectively. The coercive field at $300 \mathrm{~K}$ is greatly enhanced, compared to the bulk $(10 \mathrm{Oe})^{28}$ as well as other nanostructures. In Fig. $5 \mathrm{c}$ coercive field values at $300 \mathrm{~K}$ for bulk, nanochains, ${ }^{29,30}$ nanoparallelepipeds, ${ }^{25}$ nanoskeletons, ${ }^{19}$ nanowires, ${ }^{28}$ hyperbranches ${ }^{37}$ and nanobelts ${ }^{27}$ are presented for comparison.

\section{Conclusions}

In summary, a pseudotopotactic transformation of cobalt hydroxide nanosheets under solvothermal conditions yields single crystalline stellated cobalt metal multipods. Analysis of the intermediates indicate the transformation of hydroxide nanosheets to fcc CoO spheres and subsequently its conversion to hcp Co multipods. Due to shape anisotropy, the stellated Co multipods exhibit high coercivity in comparison to the bulk as well as other nanostructures of cobalt. This study opens up possibilities to explore $2 \mathrm{D}$ nanosheets of metal hydroxides as macromolecular precursors to synthesize nanostructures of metastable phases of metals and metal alloys.

\section{Acknowledgements}

This work was funded by DST, India (EMR/2015/001982). We thank Luana Caron for useful discussion.

\section{Notes and references}

1 V. F. Puntes, K. M. Krishnan and A. P. Alivisatos, Science, 2001, 291, 2115-2117. 
2 D. J. Sellmyer, M. Zheng and R. Skomski, J. Phys.: Condens. Matter, 2001, 13, R433-R460.

3 S. Sun and H. Zeng, J. Am. Chem. Soc., 2002, 124, 8204-8205.

4 Z. P. Liu, S. Li, Y. Yang, S. Peng, Z. K. Hu and Y. T. Qian, Adv. Mater., 2003, 15, 1946-1948.

5 Y. Wang, Q. Zhu and H. Zhang, J. Mater. Chem., 2006, 16, 1212-1214.

6 Y. Soumare, J.-Y. Piquemal, T. Maurer, F. Ott, G. Chaboussant and A. F. G. Viau, J. Mater. Chem., 2008, 18, 5696-5702.

7 C. Nethravathi, S. Sen, N. Ravishankar, M. Rajamathi, C. Pietzonka and B. Harbrecht, J. Phys. Chem. B, 2005, 109, 11468-11472.

8 J. L. Zhang, J. C. Fu, E. Q. Xie, D. S. Xue, N. J. Mellors and Y. Peng, ACS Nano, 2012, 6, 2273-2280.

9 X. Deng, D. Yang, G. Tan, X. Li, J. Zhang, Q. Liu, H. Zhang, N. J. Mellors, D. Xue and Y. Peng, Nanoscale, 2014, 6, 13710-13718.

10 R. H. Kodama, J. Magn. Magn. Mater., 1999, 200, 359-372.

11 J. I. Martín, J. Nogués, K. Liu, J. L. Vicent and I. K. Schuller, J. Magn. Magn. Mater., 2003, 256, 449-501.

12 S. Sun, C. B. Murray, D. Weller, L. Folks and A. Moser, Science, 2000, 287, 1989-1992.

13 A.-H. Lu, E. L. Salabas and F. Schüth, Angew. Chem., Int. Ed., 2007, 46, 1222-1244.

14 M. Scariot, D. O. Silva, J. D. Scholten, G. Machado, S. R. Teixeira, M. A. Novak, G. Ebeling and J. Dupont, Angew. Chem., Int. Ed., 2008, 47, 9075-9078.

15 S. Behrens, Nanoscale, 2011, 3, 877-892.

16 N. A. Frey, S. Peng, K. Cheng and S. Sun, Chem. Soc. Rev., 2009, 38, 2532-2542.

17 Y. Soumare, J.-Y. Piquemal, T. Maurer, F. Ott, G. Chaboussant, A. Falqui and G. Viau, J. Mater. Chem., 2008, 18, 5696-5702.

18 A. P. LaGrow, B. Ingham, S. Cheong, G. V. M. Williams, C. Dotzler, M. F. Toney, D. A. Jefferson, E. C. Corbos, P. T. Bishop, J. Cookson and R. D. Tilley, J. Am. Chem. Soc., 2012, 134, 855-858.

19 X. Wang, H. Fu, A. Peng, T. Zhai, Y. Ma, F. Yuan and J. Yao, Adv. Mater., 2009, 21, 1636-1640.

20 V. Tzitzios, D. Niarchos, M. Gjoka, N. Boukas and D. Petridis, J. Am. Chem. Soc., 2005, 127, 13756-13757.

21 B. Lim and Y. Xia, Angew. Chem., Int. Ed., 2011, 50, 76-85.

22 E. Ye, M. D. Regulacio, S.-Y. Zhang, X. J. Loh and M.-Y. Han, Chem. Soc. Rev., 2015, 44, 6001-6017.

23 A. P. LaGrow, S. Cheong, J. Watt, B. Ingham, M. F. Toney, D. A. Jefferson and R. D. Tilley, Adv. Mater., 2013, 25, 1552-1556.

24 M. Ohnishi, Y. Kozuka, O.-L. Ye, H. Yoshikawa, K. Awaga, R. Matsuno, M. Kobayashi, A. Takahara, T. Yokoyama, S. Bandow and S. Iijima, J. Mater. Chem., 2006, 16, 32153220 .
25 K. M. Nam, J. H. Shim, H. Ki, S.-I. Choi, G. Lee, J. K. Jang, Y. Jo, M.-H. Jung, H. Song and J. T. Park, Angew. Chem., Int. Ed., 2008, 47, 9504-9508.

26 F. Dumestre, B. Chaudret, C. Amiens, M. Respaud, P. Fejes, P. Renaud and P. Zurcher, Angew. Chem., Int. Ed., 2003, 42, 5213-5216.

27 Q. Xie, Z. Dai, W. W. Huang, J. B. Liang, C. L. Jiang and Y. T. Qian, Nanotechnology, 2005, 16, 2958-2962.

28 B. Q. Xie, Y. Qian, S. Zhang, S. Fu and W. Yu, Eur. J. Inorg. Chem., 2006, 12, 2454-2459.

29 L. Guo, F. Liang, X. G. Wen, S. H. Yang, L. He, W. Z. Zheng, C. P. Chen and Q. P. Zhong, Adv. Funct. Mater., 2007, 17, 425430.

30 Y. J. Zhang, Q. Yao, Y. Zhang, T. Y. Cui, D. Li, W. Liu, W. Lawrence and Z. D. Zhang, Cryst. Growth Des., 2008, 8, 3206-3212.

31 C. P. Gibson and K. J. Putzer, Science, 1995, 267, 1338-1340. 32 V. F. Puntes, D. Zanchet, C. K. Erdonmez and A. P. Alivisatos, J. Am. Chem. Soc., 2002, 124, 12874-12880.

33 R. Xu, T. Xie, Y. G. Zhao and Y. D. Li, Cryst. Growth Des., 2007, 7, 1904-1911.

34 L. A. T. Ngo, C. Petit and M. P. Pileni, Adv. Mater., 2001, 13, 58-62.

35 A. K. Srivastava, S. Madhavi, T. J. Whit and R. V. Ramanujan, J. Mater. Chem., 2005, 15, 4424-4428.

36 Y. J. Zhang, Y. Zhang, Z. H. Wang, D. Li, T. Y. Cui, W. Liu and Z. D. Zhang, Eur. J. Inorg. Chem., 2008, 17, 2733-2738.

37 Y. Zhang, S. W. Or and Z. Zhang, RSC Adv., 2011, 1, 12871293.

38 R. Ma and T. Sasaki, Adv. Mater., 2010, 22, 5082-5104.

39 C. Nethravathi, J. Machado, U. K. Gautam, G. S. Avadhani and M. Rajamathi, Nanoscale, 2012, 4, 496-501.

40 J. Machado, N. Ravishankar and M. Rajamathi, Solid State Sci., 2010, 12, 1399-1403.

41 C. Nethravathi, C. R. Catherine, M. Rajamathi, R. Maki, T. Mori, D. Golberg and Y. Bando, J. Mater. Chem. A, 2014, 2, 958-990.

42 C. Nethravathi, G. Harichandran, C. Shivakumara, N. Ravishankar and M. Rajamathi, J. Colloid Interface Sci., 2005, 288, 629-633.

43 R. Ma, M. Osada, L. Hu and T. Sasaki, Chem. Mater., 2010, 22, 6341-6346.

44 L. P. Zhu, H. M. Xiao and S. Y. Fu, Eur. J. Inorg. Chem., 2007, 25, 3947-3951.

45 Y. J. Zhang, S. Ma, D. Li, Z. H. Wang and Z. D. Zhang, Mater. Res. Bull., 2008, 43, 1957-1965.

46 L. Zhang and A. Manthiram, Phys. Rev. B: Condens. Matter Mater. Phys., 1996, 54, 3462-3467.

47 J. K. Yuan, K. Laubernds, Q. H. Zhang and S. L. Suib, J. Am. Chem. Soc., 2003, 125, 4966-4967.

48 D. Lin, C. A. Nunes, C. F. Majkrzak and A. E. Berkowitz, J. Magn. Magn. Mater., 1995, 145, 343-348. 